\title{
A Secondary Ion Microprobe Ion Trap Mass Spectrometer
}

\author{
Peter J. Todd and T. Gregory Schaaff \\ Chemical Sciences Division, Oak Ridge National Laboratory, Oak Ridge, Tennessee, USA
}

\begin{abstract}
An ion trap mass analyzer has been attached to an organic secondary ion microprobe. A pressure differential $>100$ can be maintained between the ion trap and microprobe. The well-focused secondary ion beam can transit a small $(2 \mathrm{~mm})$ diameter tube, but gas flow from ion trap to microprobe is impeded. This pressure differential allows the microprobe to retain imaging capability. Ion trap and microprobe data systems are integrated by taking advantage of the highly reproducible periodicity of the ion trap operating in resonant ejection mode and asynchronous signal and data acquisition afforded by commercially available interface cards. Secondary ion mass spectra and images obtained indicate an approximately 10 -fold improvement in sensitivity, although preliminary evidence indicates low $(<1 \%)$ trapping efficiency. Image data acquisition using the ion trap for mass analysis requires at least 10 times as much time compared to using a quadrupole mass filter because the mass-selected instability mode is used for mass analysis, i.e., mass resolution in the ion trap is not continuous as it is in the quadrupole. (J Am Soc Mass Spectrom 2002, 13, 1099-1107) (c) 2002 American Society for Mass Spectrometry
\end{abstract}

$\mathrm{A}$ nalytical biochemistry centers about solving three problems: (1) Compound visualization or localization, (2) separation (and pre-concentration), and (3) chemical identification. The lion's share of current research in mass spectrometry is focused on identification because mass spectrometry is sensitive, specific, and well suited to automation. A somewhat less well populated but no less enthusiastic area of mass spectrometry research involves imaging (or mapping) biologic tissue by using mass spectral methods [1]. Spatially resolved matrix assisted laser desorption/ ionization (MALDI) [2] and secondary ionization mass spectrometry (SIMS) [3] are the main tools of this effort. Imaging is accomplished by knowledge of the relative position and iterative displacement of highly focused light or high energy ion beams, respectively [1]. Separation and identification are accomplished solely by mass analysis and/or tandem mass spectrometry [4]. At least to date, the differences between imaging MALDI and SIMS are the respective molecular weights of targeted analytes and spatial resolution. MALDI is more applicable to higher molecular weight compounds such as proteins [2] and SIMS is more applicable to elements [5,6], and compounds with molecular weight less than $1000 \mathrm{Da}$ or so [3]. Organic secondary ion images display spatial resolution approaching 1

Published online August 19, 2002

Address reprint requests to Dr. P. J. Todd, Chemical Sciences Division, Oak Ridge National Laboratory, P.O. Box 2008, MS-6365, Oak Ridge, TN 37831-6365, USA. E-mail: toddpj@ornl.gov $\mu \mathrm{m}$, whereas spatial resolution with MALDI is at best, $10 \mu \mathrm{m}$.

SIMS spectra of biologic tissue tend to be complex, as do SIMS spectra of many organic compounds. Moreover, for imaging to be of any use whatsoever, a tissue sample must be a heterogeneous mixture. Below $\mathrm{m} / \mathrm{z}$ 1000 , secondary ions and fragments of them arise from many compounds and are evident at virtually every nominal $m / z$ - a feature known as "chemical noise". Although there are usually a few abundant peaks, the assumption can be made that at least a small component of even large peaks is chemical noise. Consequently, we have found tandem mass spectrometry (MS/MS) to be virtually essential for (1) determination of ion structure [7] and (2) exclusive selection of the ion structure of interest from chemical background noise [4]. To this end, we constructed an imaging secondary ion microprobe using a triple quadrupole mass filter [4]. We used this instrument to identify and map the distribution of phosphocholine ion emission under a variety of circumstances.

There are two problems with quadrupoles and mapping of tissue samples, both centering on the issue that analysis of tissue must generally be performed under static SIMS conditions [8]. Analysis and detection of secondary ions must be efficient because impact of primary ions onto the sample surface causes physical and chemical changes to the surface. Mainly as a result of the chemical changes, secondary ion emission tends to decrease with increasing primary ion dose, and mass spectra tend to become more complex. The primary ion dose at which spectra from a sample become notably 
more complex is known as the static SIMS limit [9]. A working approximation for this dose is $10^{13}$ primary ions $/ \mathrm{cm}^{2}$, although the number varies from sample to sample, and depends as well upon primary ion parameters.

Biologic molecules of interest often have fairly large molecular weights, but quadrupoles suffer from "high mass roll-off", i.e., transmission of ions with $m / z$ greater than 200 decreases rapidly [10]. Regardless of any physical apertures at the entrance of a quadrupole mass filter, ions to be transmitted through a mass resolving quadrupole must enter from within $200 \mu \mathrm{m}$ of the mean quadrupole axis; i.e., the entrance aperture of quadrupoles is approximately $200 \mu \mathrm{m}$ in diameter. This aperture decreases with higher masses [10]. A common way to mitigate roll-off is to increase ion acceleration voltage with increasing mass. This method is effective with poorly focused, or "fuzzy" ion beams, because increasing ion acceleration inherently increases the radial density of the beam. The method is effective with ion beams formed by fast atom bombardment (FAB), electron ionization (EI), chemical ionization (CI), or electrospray (ES), whose beams cannot be sharply focused.

Increasing the kinetic energy of a well-focused ion beam has a minimal effect on transmission and cannot correct mass roll-off of the secondary ion beam because the focusing optics are fixed by the geometry of the focusing electrodes. For example, organic secondary ions are emitted from a well-defined spot where the primary ion beam strikes the target. Organic secondary ions also have a very small kinetic energy spread, a few tenths of an $\mathrm{eV}$, and can thus be sharply focused, as verified experimentally with the instrument we have used [11]. With this instrument, high mass roll-off is quite evident. In fact, the losses we observe are consistent with quadrupole theory e.g., less than $10 \%$ transmission at 200 and less than $1 \%$ transmission above $\mathrm{m} / \mathrm{z}$ 500 [10]. Arguably, these numbers are much lower than those quoted by others; the difference reflects the method of measurement. An absolute measure, and the one used here, reflects the ratio of current measured without the quadrupole in place, and the measured current with the quadrupole operated in RF/DC (mass resolving mode). A more convenient method of transmission measurement is the ratio of the currents measured in RF/DC mode and RF only mode. The latter measurement also leads to a higher transmission number.

Tandem mass spectrometry with quadrupoles exacerbates the issue of static SIMS because, as conventionally practiced, MS/MS is inefficent. To be useful for MS/MS, the precursor ion must be induced to fragment into a collection of fragment ions, usually as a result of collision with some gas phase target. The intensity of only one fragment at a time can be measured, all others being lost. Very often the intensity of the most significant fragment ion is only a small fraction of the intensity of the precursor ion. With precursor ions of low intensity, a common occurrence in static SIMS, the analytical problem is not only one of chemical interference or chemical noise, but electronic noise as well. Although MS/MS is profoundly useful in reducing chemical noise, this reduction comes at the price of a decrease in electronic signal-to-noise.

One solution to the combined problems of MS/MS inefficiency and high mass roll-off is to use an ion trap as a mass analyzer $[10,11]$. Within an ion trap, MS/MS is demonstrably efficient [12]. Both precursor and fragment ions are held in the trap until they are ejected for detection. Because ion traps are efficient, it is possible to conduct so-called $\mathrm{MS}^{\mathrm{n}}$ experiments, where MS/MS is performed on precursor and then successive fragments. In contrast to quadrupole mass filters, ion traps do not suffer from discrimination of high $\mathrm{m} / \mathrm{z}$ ions. This feature makes the ion trap, operating in the appropriate mode, worth investigating as a mass analyzer for mapping biologic tissue. Ion traps are low energy (ca. 5-20 eV) mass analyzers, so that secondary ion optics appropriate for quadrupole mass analysis are appropriate for use with an ion trap. Ion traps have been used as ion mass analyzers with a number of mass spectral ionization methods, including EI, CI, atmospheric sampling glow discharge (ASGDI), ES, (non-imaging) SIMS, and MALDI. The first demonstration of effective use of ions traps with ASDGI has great significance for imaging SIMS because particularly with this ionization method, ionization must occur external to the trap and ions must be injected into it [14].

Ion traps are not necessarily the best choice for use with a secondary ion microprobe. In order to image, microprobes need high vacuum to minimize collisions between primary ions and background gas; such collisions would cause primary ion scattering. Ion traps work best under conditions of low vacuum $\left(10^{-3}\right.$ torr $)$ because collisions between ions and background are essential. To be sure, workers at Idaho Falls have constructed a very sensitive secondary ionization-ion trap instrument [13]. MALDI based ion trap systems have also been constructed both with RF multipole rods to transmit ions from the source to the ion trap while providing the space for multistage differential pumping [15], and without them [16]. For laser-based systems, the ion trap is employed because it allows MS/MS to be used for structural elucidation, mixture analysis, and chemical noise reduction.

The abundance of secondary ions can vary by orders of magnitude, and ion traps are well known to have a limited dynamic range. This prospect is not as damning for organic secondary ion emission as it would be for atomic ions. Ions emitted from compounds exhibiting low secondary ion yields are usually lost in chemical noise. On the other hand, overabundance can be corrected by reducing primary ion intensity. However, with respect to imaging, a more serious shortcoming of ion traps is the time required for mass analysis, up to 50 $\mathrm{ms} / \mathrm{spectrum}$. Typically, secondary ion images are created from some 40,000 points of a sample surface, implying a minimum of $2000 \mathrm{~s}$ for a single image. An 


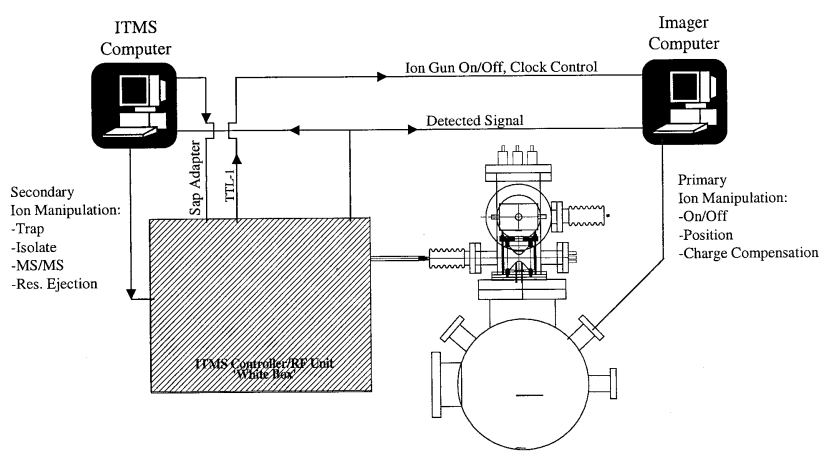

Figure 1. Schematic diagram of the ion trap microprobe.

image from the same sample based on quadrupole mass analysis would require only about 200 s [4].

Reproducibility, signal stability, and electronic noise requirements for imaging are much more demanding than they are for obtaining a mass spectrum [1]. For example, a $1 \%$ ripple in detected signal is invisible in a mass spectrum but tends to dominate an image, appearing as wavy lines. Perhaps the most significant drawback to the combination of ion trap and microprobe is that the combination is untried. In this paper, we describe our efforts to use the ion trap for secondary ion mass spectrometry and imaging, mainly of organic samples, with the goal of applying the method to biochemical analysis. In general, we limit our analysis of the system to samples which we have also analyzed using our triple quadrupole secondary ion microprobe.

\section{Experimental}

\section{Instrument Description}

Figure 1 shows a schematic diagram of the instrument. The vacuum housing consists of a 12 inch $(30 \mathrm{~cm})$ diameter stainless steel sphere featuring an array of ports fitted with high vacuum flanges. The sample introduction system (not shown) consists of a magnetically coupled sample transporter mounted to the vacuum housing via a separate introduction chamber and alignment gimbals (MDC, Hayward, CA). This system allows for introduction of any number of samples mounted on a $2 \mathrm{~cm} \times 10 \mathrm{~cm} \times 1.5 \mathrm{~mm}$ removable target. The introduction chamber is evacuated via (Welch 1402) roughing pump, which is valved off after sample evacuation. The microprobe chamber is pumped by a Balzers Model 510 Turbomolecular pump (Balzers Aktiengesellschaft, Lichtenstein). The ion trap housing is pumped differentially from the microprobe assembly by a Varian Model 969-9008 turbomolecular pump (Turin, Italy).

Figure 2 shows a schematic diagram of the aperture permitting differential pressure between ion trap and secondary ion source housings. Primary and secondary ion optics are essentially identical to those of the triple quadrupole microprobe system [17]. Key features of this system include digital dynamic emittance matching

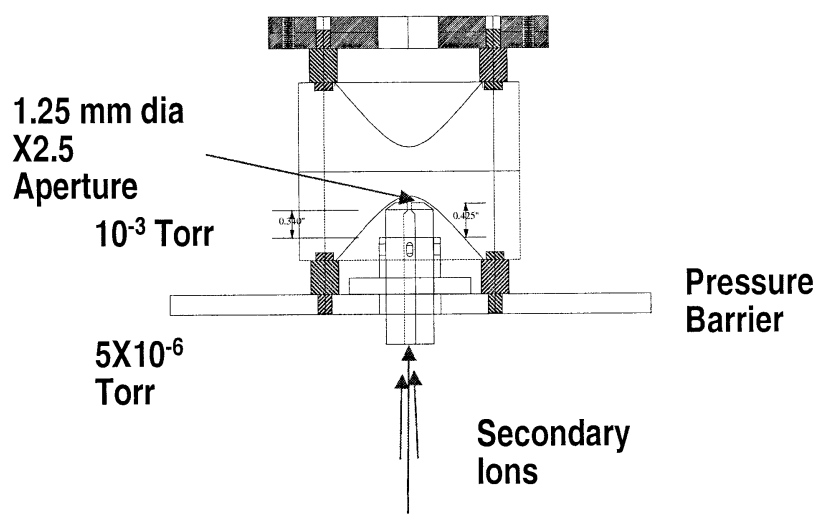

Figure 2. Aperture inserted between microprobe and ion trap permits a pressure differential of about 100 between the two regions, obviating the need for RF multipole ion guides. The minimum diameter of the aperture is determined by how well the secondary ion beam can be focussed.

(DEM) [18] by which the secondary ion optics are changed as the primary ion beam moves from point to point on the sample. In general, mass spectrometers will efficiently transmit a beam of ions a few hundred microns in diameter. By adjusting deflectors within the secondary ion source in consonance with the position of the primary ion beam spot, secondary ions from large areas of the sample can be extracted, focused, and transmitted through the mass spectrometer. The tubular aperture of the design takes advantage of the well focused and narrow ( $<200 \mu \mathrm{m}$ diameter) secondary ion beam to provide a pressure differential of about $10^{2}$ between microprobe and ion trap vacuums, as expected. In fact, we observe from Bayard-Alpert gauges in the two respective regions a helium pressure differential of 200. With a $\mathrm{Cs}^{+}$primary beam, a pressure of $10^{-3}$ torr in the ion trap region has no discernable effect on the spatial resolution of about $5 \mu \mathrm{m}$ evident in total ion images.

Crucial to operation of the ion trap microprobe is coordination of ion trap electronics with microprobe electronics. Electronics of the microprobe are described elsewhere but essentially consist of manual high voltage (ca. $10 \mathrm{kV})$ primary ion acceleration power supplies and computer controlled primary ion raster, beam on/off and secondary ion detection electronics [17, 19]. Software to control the microprobe is written in Visual Basic, using National Instruments (Austin, TX) ATMIO16X and AO-10 interface cards and drivers running under Microsoft Windows 3.11. Importantly, this software system allows primary ion position and secondary ion detection to be controlled independently of each other by externally applied synchronization (TTL) pulses. A further feature of the system is coordination between position of mouse cursor on the displayed secondary ion image and the location of the primary ion beam on the sample. We refer to the computer and interface controlling the microprobe as the imager computer.

Electronics of the ion trap are essentially those of the 


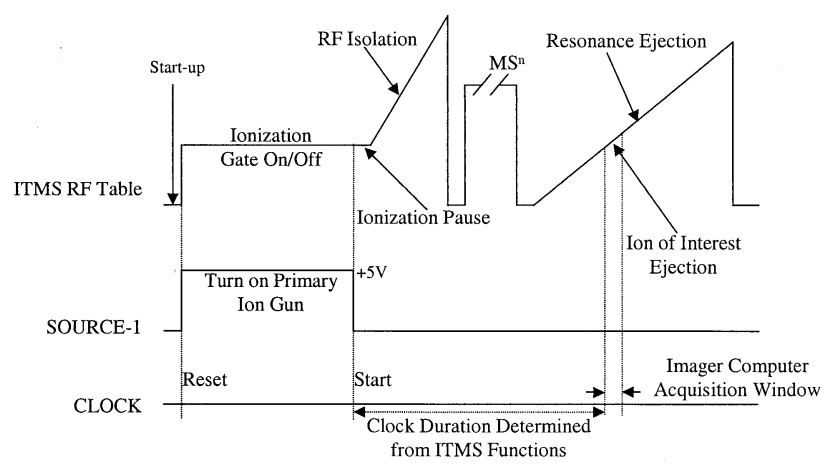

Figure 3. Connections between ion trap and microprobe data systems are centered about the ITMS TTL outputs and the National Instruments interface card. The former emits either edges or pulses indicating the beginning of an ion trap operation; the latter detects the signal, and after appropriate delay, measures the signal at the detector.

ITMS, operating with ICMS software (ThermoFinnigan MAT, San Jose). The interconnection and flow of data in the interface between ion trap and microprobe is shown schematically in Figure 1. Relevant operations are (1) "Ionization", (2) ion manipulation (e.g., trapping, isolation, MS/MS), (3) resonant ejection, and (4) detection. Parameters of these operations are transmitted from the ITMS computer to the Scan/Acquisition Processor (SAP). The fact that parameters are loaded before operations are performed allows the data to be acquired asynchronously to the (ISA) data bus of the ITMS computer. Consequently, the suite of operations is performed in a precise periodic waveform, independent of any latency of the ITMS computer. The sequence of these operations is indicated by Figure 3, top trace, along with a stylized representation of the RF amplitude applied to the ring of the ion trap.

Importantly, the SAP transmits a HIGH output $(+5$ V) for a period indicated as "Ionization" in Figure 3. We connected this ON/OFF signal directly to the (SOURCE-1) input of the ATMIO16X interface card in the imager computer. The leading edge of this (TTL1) waveform is used to turn on the primary ion beam. The primary ion beam is turned off after a variable delay specified by user input to the imager computer, but the variable delay must be at least $2 \mathrm{~ms}$ less than the Ionization period specified in the ITMS software, as indicated in Figure 1. Secondary ions require about 1-2 $\mathrm{ms}$ to transit from sample to ion trap; by extending the Ionization period of the ITMS $2 \mathrm{~ms}$, the RF level is maintained, permitting trapping.

Although the primary ion gun is synchronized to the Ionization period of the ITMS electronics, mass spectra are acquired and stored using the ITMS data system. For a single mass spectrum, a $1 \mathrm{~ms}$ primary $(10 \mathrm{keV} 8$ $\mathrm{nA}) \mathrm{Cs}^{+}$pulse and $3 \mathrm{~ms}$ Ionization period is followed by mass analysis at the fixed scan rate $(5555 \mathrm{Da} / \mathrm{s})$ under resonance ejection conditions $\mathrm{q}_{\mathrm{z}}=0.9$.

Acquisition of a selected secondary ion image requires that imager and ITMS data systems work to- gether. Processed ion signal from the SAP (after current-to-voltage conversion and amplification) is sampled by the high impedance instrumentation amplifier of the imager computer's ATMIO16X interface card. Resonance ejection of a specific ion is synchronized and its intensity measured by the imager computer as follows. When the falling edge of the ionization trigger is detected by the ATMIO16X, its CLOCK is used to delay a voltage measurement. This delay is determined by the duration of ion manipulation steps (e.g., isolation, MS/MS, etc). After this delay, the voltage is measured for a fixed period of a few hundred $\mu \mathrm{s}$, corresponding to the width of one Da; the accuracy of this duration is routinely verified by external oscilloscope. For example, a sampling period of $2.0 \mathrm{~ms}$ is used with an ITMS fixed scan rate $=1.8 \mathrm{~ms} / \mathrm{Da}$. In this manner, the user can monitor the ion abundances on the ITMS data system while simultaneously monitoring the progress of the resulting image being generated by the Imager Computer. This type of timing scheme also allows for the Imager Computer to measure the intensity of multiple ions that are ejected from the ion trap. The steps involved in obtaining a selected secondary ion image are: (1) Ionization (primary ion beam on/off, ion transit delay); (2) all ion manipulation steps (trapping, isolation, MS/MS), which determine delay duration, and (3) acquisition of signal intensity from a single or multiple ions during resonance ejection. This process is repeated as necessary at each of 40,000 points on a sample surface.

\section{Instrumental Conditions}

Table 1 contains a range of primary and secondary ion conditions and parameters used to acquire the data presented here. Charge compensation was not used. During operation, an indicated pressure of $10^{-4}$ torr He was maintained in the ion trap. This indicated pressure corresponds to a pressure of $10^{-3}$ torr He when corrected for the Bayard-Alpert type ion gauge calibration with $\mathrm{N}_{2}$ [20]. The base pressure of the instrument, i.e., before introduction of any gas, is $\sim 8 \times 10^{-8}$ torr.

\section{Samples}

Separate solutions of alkali halides $(\mathrm{KCl}, \mathrm{RbCl}$, and $\mathrm{CsCl}$, all analytical reagents), choline chloride (Aldrich), phosphatidylcholine dipalmitate (Aldrich), were prepared immediately before being spotted $(0.3 \mu \mathrm{L})$ onto copper targets and allowed to dry in air. Samples of human fingerprints were prepared by rubbing the index finger across the forehead and touching the stainless steel target with the finger [21].

\section{Results and Discussion}

The easiest test of any secondary ion mass spectrometer is to obtain the mass spectrum from a salt mixture. Alkali halides, in particular, yield abundant positive 
Table 1. Instrumental parameters

\begin{tabular}{ll}
\hline Parameter & Value \\
\hline \hline Microprobe static pressure & $10^{-8}$ torr \\
Microprobe operating pressure He & $5 \times 10^{-5}$ torr \\
lon trap operating pressure He & $10^{-3} \mathrm{torr}$ \\
Primary ion species & $\mathrm{Cs}^{+}$ \\
Primary ion kinetic energy & $10 \mathrm{keV}$ \\
Primary ion current (continuous) & $(.25-8 \mathrm{nA})$ as indicated \\
Primary ion spot diameter & $50 \mu \mathrm{m}$ \\
Primary ion current density & $125 \mu \mathrm{A} / \mathrm{cm}^{2}-4 \mathrm{~mA} / \mathrm{cm}^{2}$ \\
Primary ion dose/image (1 ms/pixel) & $1.5-48 \times 10^{7} \mathrm{ions} / \mathrm{cm}^{2}$ \\
Primary ion dose/mass spectrum (1 ms) & $1.5-48 \times 10^{7} \mathrm{ion} / \mathrm{cm}^{2}$ \\
Primary ion duty cycle (on/period) & $(1 \mathrm{~ms}) /(20-50 \mathrm{~ms})$ \\
Raster size & $1 \mathrm{~cm} \times 1 \mathrm{~cm}$ \\
Image acquisition time (200 $\times 200)$ & $2000 \mathrm{~s}-20,000 \mathrm{~s}$ \\
Secondary ion acceleration energy & $12 \mathrm{eV}$ \\
Conversion dynode potential (- secondary ions) & $+12 \mathrm{kV}$ \\
Multiplier potential & $-2.3 \mathrm{kV}$ \\
Image data acquisition rate (time/pixel) & $20-50 \mathrm{~ms}$ \\
Samples/pixel & $1-10$ \\
Sample area/pixel & $50 \mu \mathrm{m} \times 50 \mu \mathrm{m}$ \\
\hline
\end{tabular}

secondary ions that are easy to identify, and the abundance does not seem to be dependent upon any matrix effects. Figure 4 shows the mass spectrum obtained from an aqueous solution containing equal concentrations of $\mathrm{KCl}, \mathrm{RbCl}$, and $\mathrm{CsCl}$, placed on a target and allowed to dry. This mass spectrum reflects the sum of 20 spectra, each obtained following $1 \mathrm{~ms}$ of primary ion irradiation. Although secondary ion yields from alkali salts are typically high, and often assumed approximately unity, analysis by ion traps for these ions cannot be assumed to be high for two reasons. First, atomic secondary ions generally have a broad range of kinetic energy, with maxima in the range of $20 \mathrm{eV}$ and tails up to $200 \mathrm{eV}$ [8]. This feature makes them impossible to

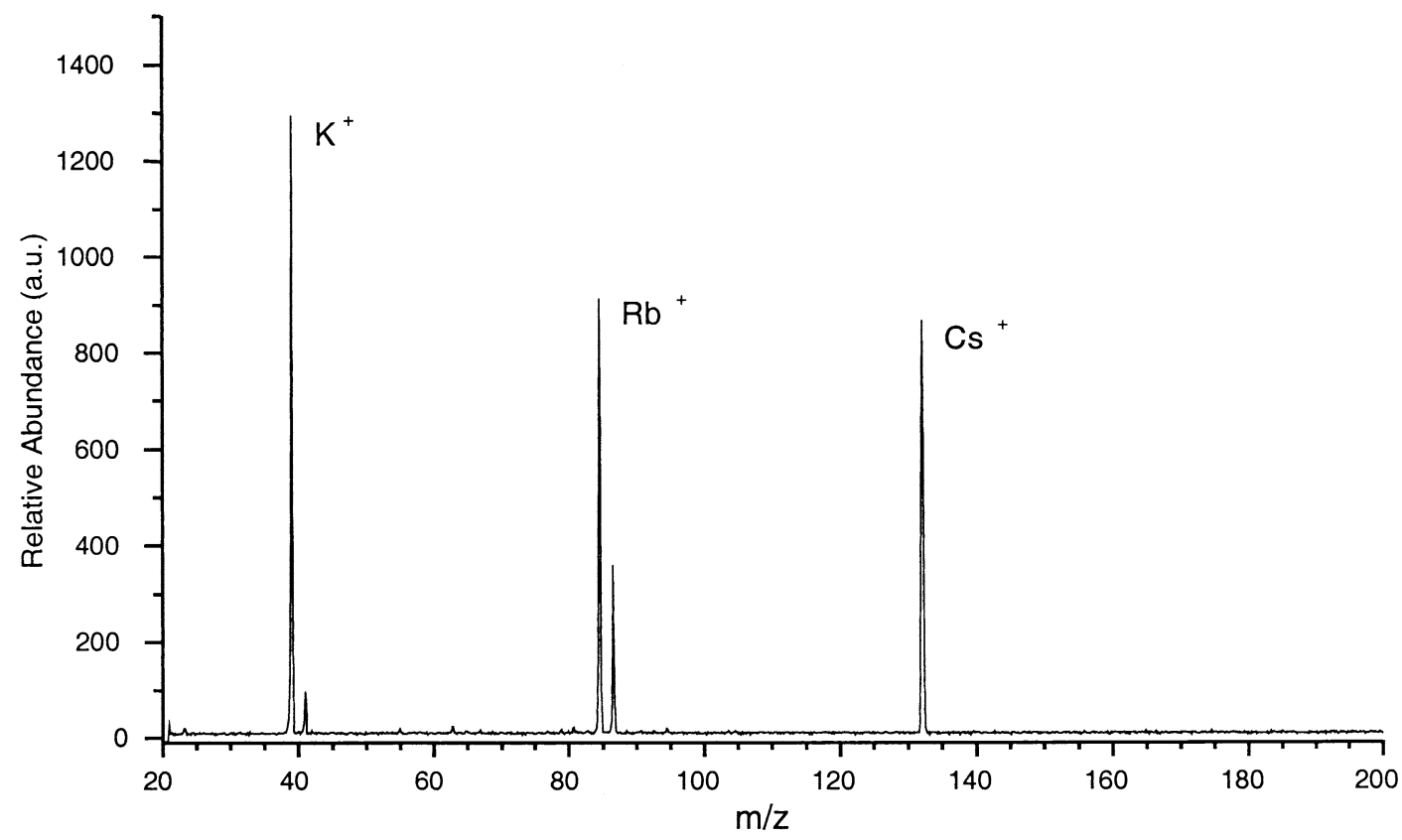

Figure 4. The sum of 20 mass spectra obtained from a single point on a sample of a $\mathrm{KCl}, \mathrm{RbCl}$, and $\mathrm{CsCl}$ salt mixture demonstrate the general sensitivity of the ion trap microprobe. Although alkali halides generally yield abundant secondary ions, atomic ions are much less likely to be trapped than molecular secondary ions. About $10^{9}$ primary ions were used to generate this summed spectrum from one spot on the sample. The absence of organic secondary ions in this spectrum is notable, and reflects the facts that (a) atomic ions have fairly large kinetic energy distributions and (b) dynamic emittance matching causes secondary ions to travel a tortuous path, which is determined by deflector voltages and ion kinetic energy. When tuned for atomic ions, polyatomic secondary ions are preferentially lost because they have very small initial kinetic energy. 


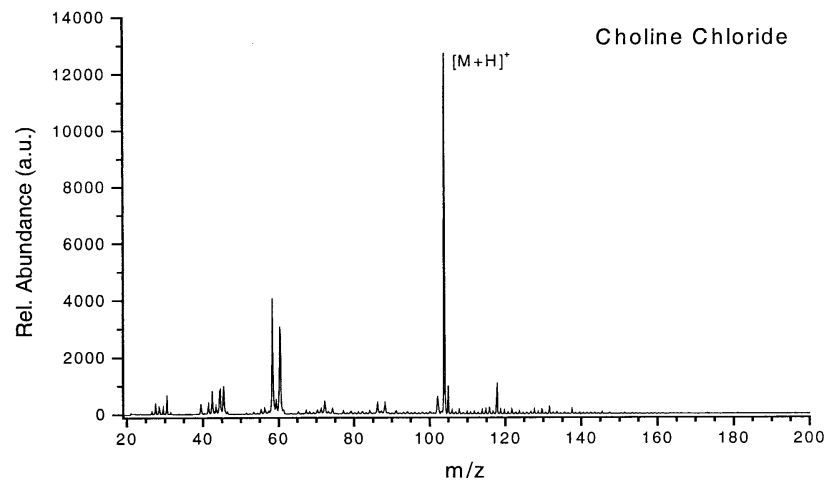

Figure 5. Choline chloride spectrum is used to tune up the mass spectrometer, since neither the spectrum nor the total secondary ion current varies with primary ion dose. The peak at $m / z 118$ is known to be an impurity of choline chloride arising from oxidation.

focus well, especially for a low energy analyzer like an ion trap or quadrupole mass filter. Secondly, monatomic ions in general, and alkali ions in particular, have low cross sections for collisions with He. In fact, preliminary measurements made of the ions transmitted through the ion trap indicate much less than $1 \%$ trapping efficiency. Such measurements were made by subtraction of the detector current made with the ion trap operating, from the detector current measured with all the electrodes of the ion trap grounded. This measurement compares unfavorably with simulation studies, where a trapping efficiency of $4 \%$ is estimated for polyatomic $m / z 106$, and $25 \%$ for polyatomic $\mathrm{m} / \mathrm{z}$ 1522 ions [21].

A spectrum more relevant to organic SIMS is shown in Figure 5, obtained from choline chloride. This compound possesses the unusual feature that even after prolonged irradiation by primary ions, the secondary ion intensity and mass spectra remain invariant. The spectrum is marked by abundant $m / z 104$, choline, with major rearrangement fragment ions evident at $m / z 58$ and 60 . The peak at $m / z 118$ is the result of oxidation and is a common impurity of choline chloride. This mass spectrum is virtually identical to those obtained using other mass analyzers.

Electroformed grids are often used as targets to provide proof that an imaging instrument is working properly but such grids are necessarily metallic, not organic. To establish the utility of the ion trap microprobe, "choline" ink was prepared by dissolving choline chloride in methanol. A recognizable pattern of the ink was then written on a $\mathrm{Cu}$ target, using a syringe tip. This process was somewhat frustrated by the tendency of methanol to run on the smooth surface of $\mathrm{Cu}$. After the ink dried, the $m / z 104$ intensity was mapped using the microprobe. The result is shown in Figure 6, where the handwritten letters "ORNL" are clearly visible, if somewhat distorted. A period of about 20 min was required to obtain this image; a similar image using a quadrupole system would require about $2 \mathrm{~min}$. Three

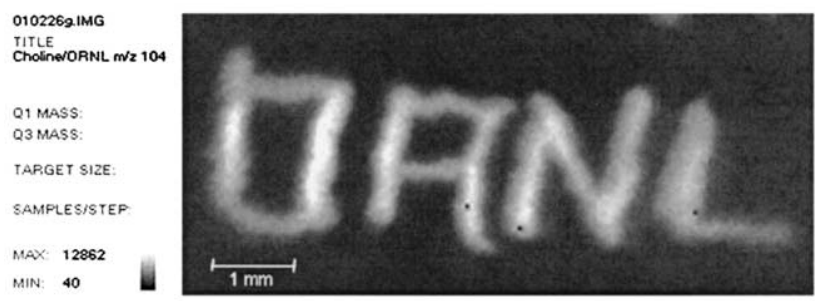

Figure 6. Secondary ion image $(\mathrm{m} / \mathrm{z} 104)$ obtained from ink prepared from choline chloride and methanol. The coarse nature of the lettering results from spreading of the ink and the small (ca. $1 \mathrm{~mm}$ ) letter size. The three small black dots are a result of timing errors (glitches) between the microprobe and ion trap due to occasional arcs of the primary ion gun.

dark pixels are evident on each of the last three letters. These arise from "glitches" or timing errors between ion trap and microprobe electronics. The source of these glitches appears to be RF emission from the primary ion source caused by, for example, arcing or by some other source, such as one of the computers. In any case, the glitches are occasional and tend to appear, if at all, in different parts of the images obtained from replicate samples.

One essential feature is the ability to perform MS/ MS. In Figure 7, we show the MS/MS spectrum of the $\mathrm{m} / \mathrm{z} 184$ phosphocholine secondary ion obtained from a sample of phosphatidylcholine dipalmitate. This MS/MS spectrum is the result of collisional activation of $m / z 184$ for a period of $20 \mathrm{~ms}$ at $\mathrm{q}_{\mathrm{z}}=0.2(78 \mathrm{kHz})$ with resonance excitation amplitude of $10 \mathrm{mV}$. As we previously reported, the MS/MS spectrum of phosphocholine is marked by abundant $m / z 86$, reflecting the loss of the elements of phosphoric acid, $\mathrm{H}_{3} \mathrm{PO}_{4}$. Evident in this spectrum is improved signal-to-noise and better mass resolution than we observed when using the triple quadrupole system.

Negative secondary ion emission is important because fatty acids and many phospholipids show abundant and diagnostic secondary anion emission. For example, Figure 8 shows the fatty acid region of mass spectrum taken from a fingerprint. Palmitic and pamitoleic anions $(\mathrm{m} / \mathrm{z} 255$ and 253, respectively) dominate the spectrum, although an abundant peak is also found corresponding to oleic anion $(\mathrm{m} / \mathrm{z} 281)$. This spectrum is unremarkable in that the anions observed are those expected, but we found the sensitivity of the ion trap to be quite remarkable in that the spectrum could be obtained from a single spot on an entire fingerprint. We have found spectra obtained from other volunteers to show variation in the relative abundance of these three characteristic secondary ions. No biomedical or forensic significance should be attributed to this observation without a larger set of data.

The fact that a SIMS spectrum can be obtained from a single fingerprint suggests that a secondary ion image of characteristic anions may be quite feasible. Figure 9 shows that a secondary ion image of a fingerprint can be readily obtained and identified. This image was 


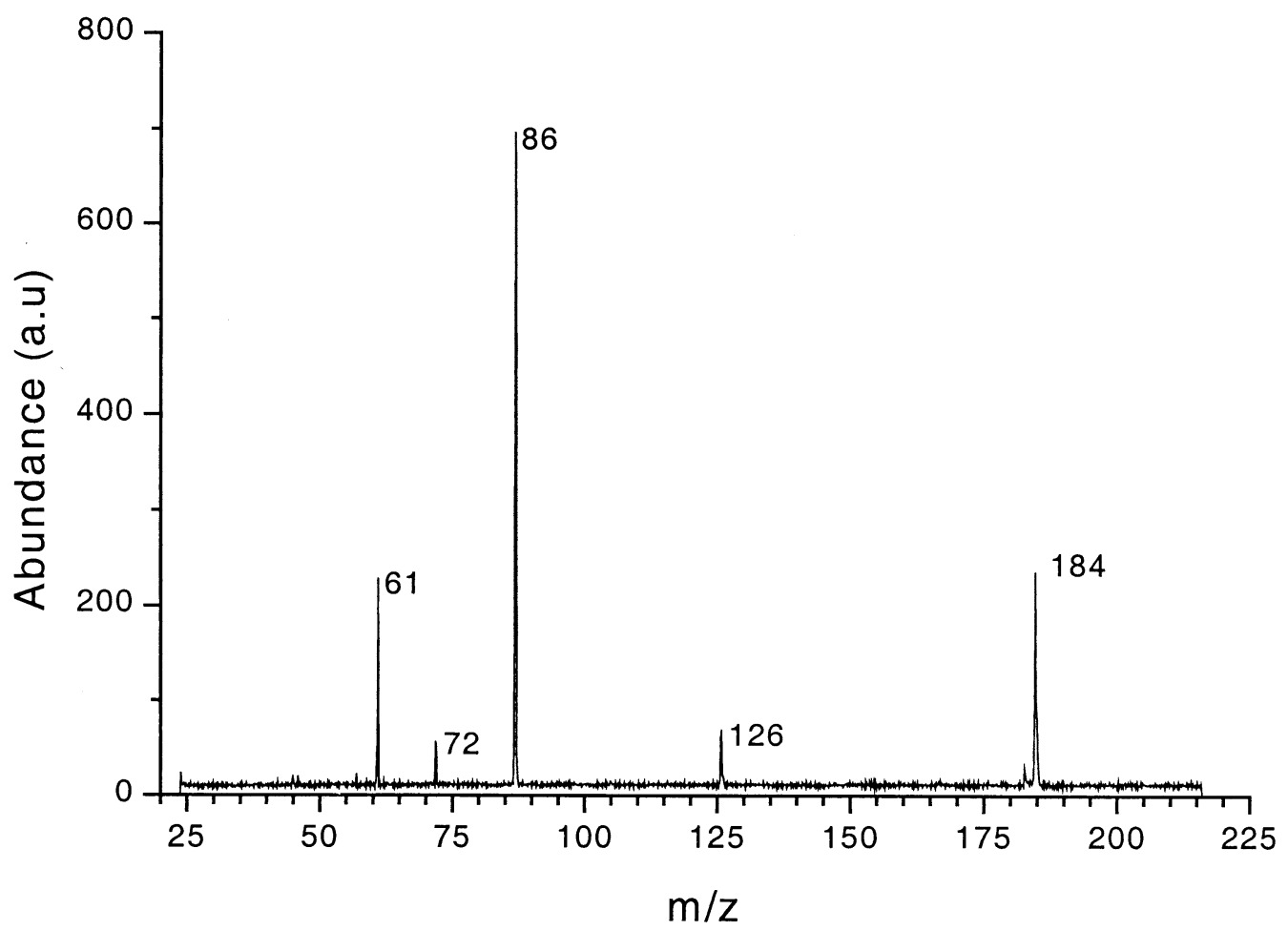

Figure 7. MS/MS spectrum obtained from the $\mathrm{m} / \mathrm{z} 184$ (phosphocholine) secondary ion emitted from phosphatidylcholine dipalmitate.

acquired over a period of $2 \mathrm{~h}$, again reflecting the long period of time required to perform imaging using an ion trap. With our previous quadrupole based system, we could scan the area of the fingerprint in about 2 min; it is arguable that the quality of the image would be sufficient for any analytical purpose. The image shown

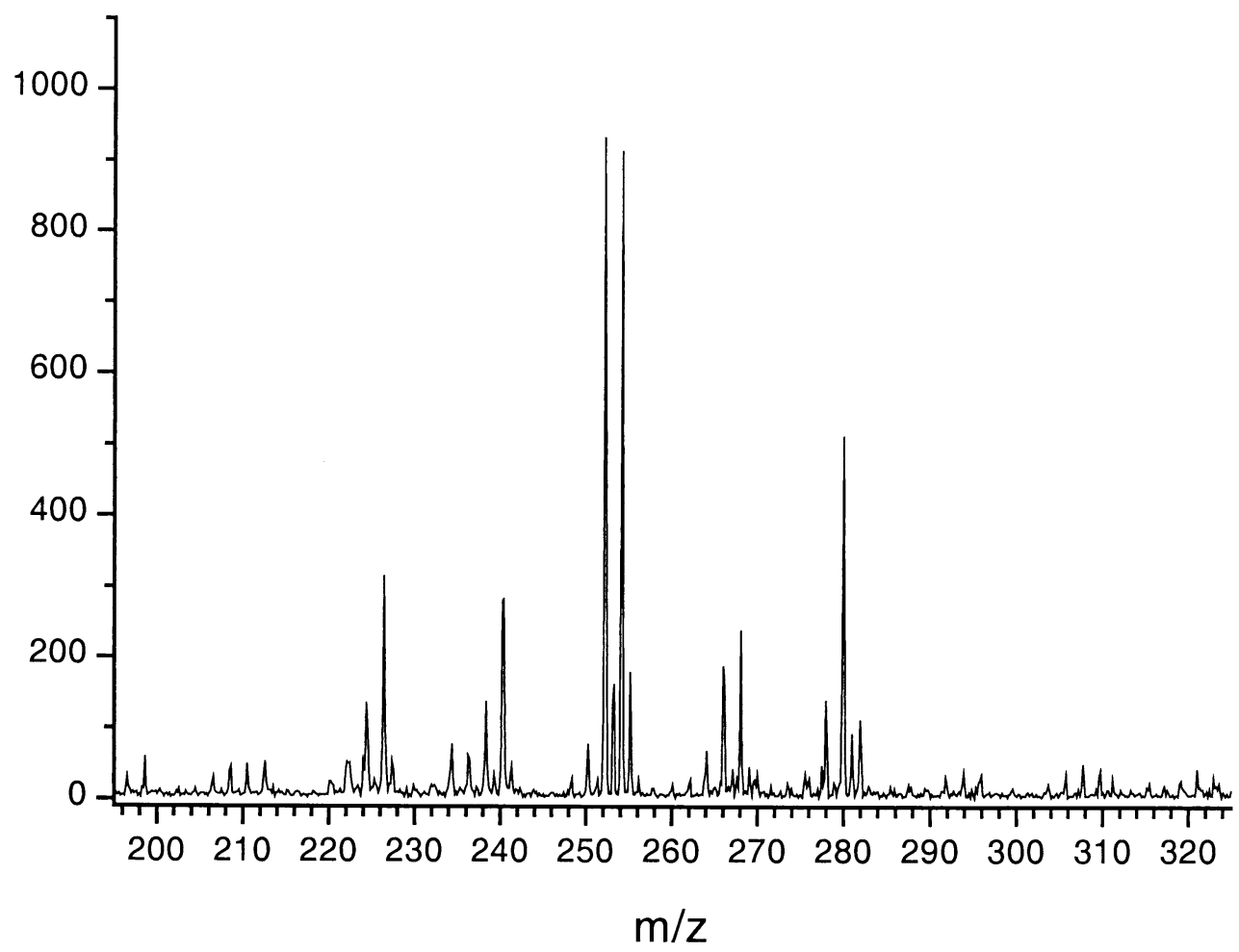

Figure 8. Negative secondary ion mass spectrum taken from the fingerprint of one of the authors. 


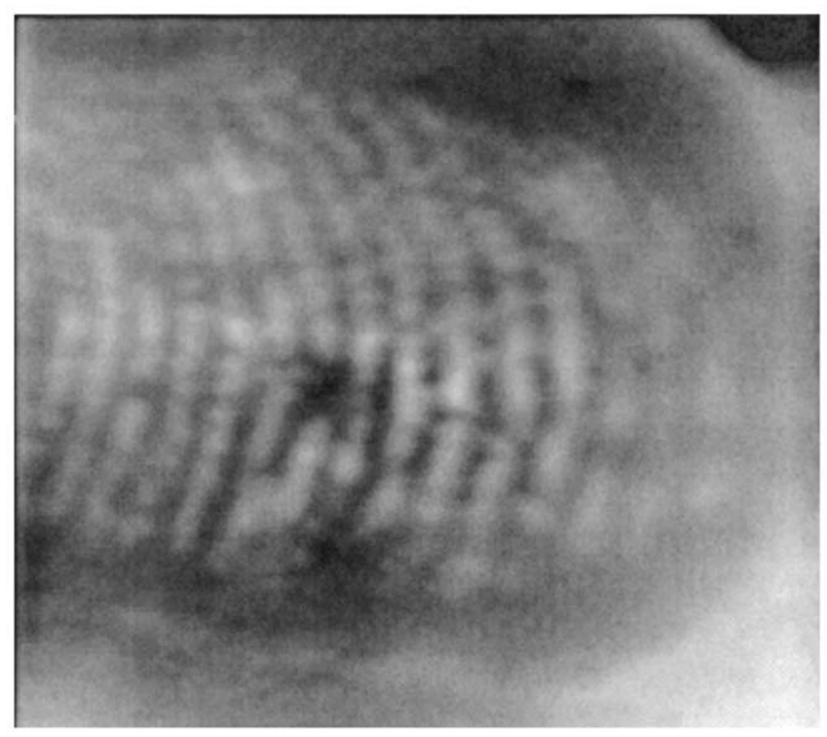

Figure 9. Secondary ion image $(\mathrm{m} / \mathrm{z} 255)$ of the fingerprint used to obtain the spectrum shown in the previous figure demonstrating a field of view of $1 \mathrm{~cm}$. This image required over $2 \mathrm{~h}$ to acquire.

in Figure 9 also answers questions about the noise that might be introduced by the use of an ion trap. To the extent we are able to see, the repeatedly swept waveforms of the ion trap are sufficiently reproducible as to produce no evident noise or ripple in the image.

The larger significance of the fingerprint image is peculiar to secondary anion emission from biologic tissue. We have attempted to obtain anion images from tissue samples as thin as $20 \mu \mathrm{m}$, but these samples build up a substantial positive charge during analysis, preventing a sensible analysis. However, no evidence of charging was observed in analyzing the fingerprint. Thus, we suggest that simply touching a tissue sample to the target, removing it, and then analyzing the residue can yield secondary anion images of tissue. This approach is currently being investigated in our system, but has been used by MALDI-based systems [1].

\section{Conclusions}

In general, we found the ion trap microprobe to be about an order of magnitude more sensitive than our previous triple quadrupole system. We anticipate this sensitivity can be improved further by increasing trapping efficiency with pulsed trap electronics and collision gas valves. On the other hand, the ion trap's sensitivity comes at the expense of time, requiring approximately ten times as long to obtain a secondary ion image. An advantage that accrues to secondary ion mass spectrometry is that the primary ion beam can be removed from the sample during analysis so that sample is not lost during the time required for mass analysis. Reproducibility of the ion trap as a mass analyzer is quite adequate. That is, the images show no evidence of ripple or other noise phenomena that would be an indication of even minute shifts in ion trap waveform generation. The fact that sparkinduced glitches are so noticeable in Figure 6 is testimony to the sensitivity of images to any weakness in the train of data acquisition.

Evidence from measurements of the trapping efficiency of atomic secondary ions indicate some improvement is necessary, especially to trap ions at higher mass. Atomic ions display small cross section for collision with He; higher molecular weight ions display a much larger cross section, but also carry much greater momentum, and consequently must suffer a greater number of collisions for trapping to occur. We intend to make improvements to trapping efficiency by (1) use of pulsed valves and (2) electronic control of the ion trap's end caps.

\section{Acknowledgments}

The authors are indebted to S. P. Markey and H. Fales of the National Institutes of Health for donation of major ion trap components. Similary, M. B. Wise and S. A. Lammert of ORNL kindly loaned them additional components necessary for operation of the instrument. They gratefully acknowledge generous financial support for biomedical applications of SIMS from the National Institutes of Health (grant GM 41617) and for support for basic research into SIMS, from the U.S. Department of Energy, Office of Research, sponsored by the Division of Chemical Sciences, Geosciences, and Biosciences, Office of Basic Energy Sciences, U.S. Department of Energy, under contract DE-AC05-00OR22725 with Oak Ridge National Laboratory, managed and operated by UT-Battelle, LLC.

\section{References}

1. Todd, P. J.; Schaaff, T. G.; Chaurand, P.; Caprioli, R. M. J. Mass Spectrom. 2001, 36, 355-369.

2. Caprioli, R. M.; Farmer, T. B.; Gile, J. Anal. Chem. 1997, 69, 4751-4760.

3. Todd, P. J.; McMahon, J. M.; Short, R. T.; McCandlish, C. A. Anal. Chem. 1997, 69, 529A-535A.

4. Todd, P. J.; Grimm, C. C.; Holland, W. M.; Markey, S. P. Anal. Chem. 1992, 64, 1871-1878.

5. Burns, M. S. J. Microsc. 1982, 127, 237-258.

6. Chandra, S.; Morrison, G. H. Biol. Cell 1992, 74, 31-42.

7. McMahon, J. M.; Short, R. T.; McCandlish, C. A.; Brenna, J. T.; Todd, P. J. Rapid Commun. Mass Spectrom. 1996, 10, 335-340.

8. Benninghoven, A. Secondary Ion Mass Spectrometry Basic Concepts, Instrumental Aspects, Applications, and Trends. WileyInterscience: New York, 1987, 664-689.

9. Benninghoven, A. Surf. Sci. 1971, 28, 541.

10. Dawson, P. H. Quadrupole Mass Spectrometry and Its Applications. Elsevier: Amsterdam, 1976, 105.

11. Short, R. T.; Grimm, C. C.; Todd, P. J. J. Am. Soc. Mass Spectrom. 1991, 2, 226-231.

12. March, R. Quadrupole Storage Mass Spectrometry. John Wiley: New York, 1989, 369-70.

13. Ingram, J. C.; Groenewold, G. S.; Appelhans, A. D.; Dahl, D. A.; Delmore, J. E. Anal. Chem. 1996, 68, 1309-1316.

14. McLuckey, S. A.; Glish, G. L.; Asano, K. G. Anal. Chim. Acta 1989, 225, 25-35.

15. Qin, J.; Chait, B. T. Anal. Chem. 1996, 68, 2102-2107. 
16. Troendle, F. J.; Reddick, C. D.; Yost, R. A. J. Am. Soc. Mass Spectrom. 1999, 10, 1315-1321.

17. Grimm, C. C.; Short, R. T.; Todd, P. J. J. Am. Soc. Mass Spectrom. 1991, 2, 362-371.

18. Liebl, H. U. S. Patent 3,517,191 (1965).
19. Short, R. T.; McMahon, J. M.; Holland, W. M.; Todd, P. J. J. Am. Soc. Mass Spectrom. 1994, 5, 37-43.

20. Bartmess, J. E.; Georgiadis, R. M. Vacuum 1983, 33, 149.

21. Quarmby, S. T.; Yost, R. A. Int. J. Mass Spectrom. 1999, 190-191, 81-102. 